\title{
Output Feedback Stabilization of a Class of Non-affine Nonlinear Systems in Discrete Time
}

\author{
Zhenfeng Chen \\ College of Automation \\ Guangdong Polytechnic Normal University \\ No. 293, Zhongshan Avenue, Tianhe District \\ Guangzhou 510665, China
}

\author{
Zhongsheng Wang \\ Guangdong Polytechnic \\ Normal University \\ Guangzhou 510665, China
}

\author{
Jian Cen \\ Guangdong Polytechnic \\ Normal University \\ Guangzhou 510665, China
}

\begin{abstract}
In this paper, output feedback control is investigated for a general class of uncertain non-affine nonlinear systems in discrete time. Control system design employs feedback linearization, coupled with a novel filter which is built to estimate the feedback linearization error. Output feedback control is then developed to stabilize the systems by utilizing the estimation. In the control design, implicit function theorem and the mean value theorem are exploited to handle the difficulty of non-affine appearance of the control input. The proposed control is of great significance in engineering practice due to its linear control architecture, high dynamic performance, clear physical meanings and robustness to the modeling errors.
\end{abstract}

\section{General Terms:}

Algorithms, Nonlinear Control Theory

\section{Keywords:}

Nonaffine nonlinear systems, discrete-time systems, output feedback control, uncertainty

\section{INTRODUCTION}

Recently, the control problem of non-affine nonlinear systems has attracted increasing research interests within the control systems community. Many elegant control schemes for continuous-time systems in this area have been obtained, including adaptive neural network (NN) control [1]-[4], adaptive fuzzy control [5], and backstepping control by incorporating the adaptive $\mathrm{NN}$ control method [6]. However, due to the the fact that the linearity property of the derivative of a Lyapunov function in continuous-time is not presented in the difference of the Lyapunov function in discrete time [7] and the lack of applicability of Lyapunov techniques [8], many elegant control methods in continuous-time domain may be not suitable for discrete-time systems. Therefore, it is challenging and very important to develop control scheme for discrete-time nonlinear systems.

In [9], the authors considered the control problem of a class of nonlinear discrete-time systems with general relative degree and proposed a stable NN controller through backpropagation such that the closed loop achieved the desired control performance. For strict- feedback nonlinear dynamical systems in discrete time, adaptive $\mathrm{NN}$ control schemes were presented by state feedback in [10] and output feedback in [11]. And for pure-feedback nonlinear systems in the discrete-time form adaptive neural output feedback tracking controllers were further investigated in [12, 13]. The common features of these adaptive control approaches are employed online NN to compensate for the unknown continuous functions, of which the tremendous advantage is that the unknown functions can be approximated to an arbitrarily accuracy. A limitation lies that reconstruction errors must occur if the structure of the approximators (i.e., the number of hidden layers and neurons of $\mathrm{NN}$ ) is not sufficiently rich, and these reconstruction errors are introduced into the closedloop and deteriorate the performance of the system. In general, the richer the structure is the more favorable performance the system exhibits. However, the richer structure may lead to the complexity of the controller and cause the heavy computational burden.

In practical applications, it is expected that control strategy can reduce the effects of uncertainties to a given accuracy, and has a simple control architecture, high dynamic performance and clear physical meanings. In this paper, output feedback control is investigated for a general class of highly uncertain discrete-time nonaffine nonlinear systems. Control system design employs feedback linearization, coupled with a novel filter which is built to estimate the feedback linearization error. Output feedback control is then developed to stabilize the systems by utilizing the estimation. To handle the difficulty of non-affine appearance of the control input, implicit function theorem and the mean value theorem are exploited in the control design. The main contributions of the paper are as follows:

- Novel filter is constructed for a class of non-affine nonlinear systems, by which the feedback linearization error can be estimated without using $\mathrm{NN}$;

- Based on the novel filter, robust output feedback control is developed to stabilize the discrete-time non-affine nonlinear systems;

- The proposed control is of great significance in engineering practice due to its linear control architecture, high dynamic performance, clear physical meanings and robustness to the modeling errors.

Throughout this paper, $\mathbb{Z}_{0}^{+}$stands for nonnegative integers, $|\cdot|$ denotes the absolute value of a scalar, $\|\cdot\|$ denotes the norm of a matrix, $I_{n \times n}$ denotes the $n \times n$ dimension identity matrix, 
$a:=b$ means that $b$ is defined as $a,[]^{T}$ denotes the transpose of a vector/matrix, $\sigma(M)$ denotes the spectral radius of a square matrix $M$, i.e., $\sigma(M):=\max _{1 \leq i \leq n}\left\{\left|\lambda_{i}\right|\right\}$, where $\lambda_{1}, \cdots, \lambda_{n}$ are the eigenvalues of matrix $M, A:=\left[\begin{array}{ccc}0 & & \\ \vdots & I_{(n-1) \times(n-1)} & \\ 0 & \cdots & 0\end{array}\right]_{n \times n}$ $B:=[0,0, \cdots, 0,1]_{n}^{T}, C:=[1,0, \cdots, 0,0]_{n}^{T}$. The following lemma will be used for control design and stability analysis in Section 3.

LEMMA 1 IMPLICIT FUNCTION THEOREM[14]. Assume that function $f(x, u): \mathbb{R}^{n} \times \mathbb{R} \rightarrow \mathbb{R}$ is continuously differentiable $\forall(x, u) \in \mathbb{R}^{n} \times \mathbb{R}$. If there exists a constant $c>0$ such that $\partial f / \partial u \geq c>0, \forall(x, u) \in \mathbb{R}^{n} \times \mathbb{R}$, then, there exists a continuous function $u(x): \mathbb{R}^{n} \rightarrow \mathbb{R}$ such that $f(x, u(x))=0$

\section{PROBLEM FORMULATION}

Consider the non-affine nonlinear systems in the following form

$$
\begin{cases}y_{k+n} & =f\left(x_{k}, u_{k}\right), k \in \mathbb{Z}_{0}^{+} \\ x_{k} & =\left[y_{k}, y_{k+1}, \cdots, y_{k+n-1}\right]^{T}\end{cases}
$$

where

$u_{k} \in \mathbb{R}$ is the control input;

$y_{k} \in \mathbb{R}$ is the measured output;

$x_{k} \in \mathbb{R}^{n}$ is the state vector;

$f: \mathbb{R}^{n+1} \rightarrow \mathbb{R}$ is an unknown implicit function;

$\psi: u_{k} \rightarrow f$ is a bijection for every fixed $\left(x_{k}, u_{k}\right)$;

$n \geq 1$ is system order

Assume that system function $f$ is continuously differentiable with respect to all the arguments and only the output $y_{i}, 0 \leq i \leq k$ are available for feedback at each step $k$. And without lose of generality, assume that the origin $x=0$ is an equilibrium point of system (1), i.e., $f(0,0)=0$. In order to design a control input $u$ which stabilizes the system, the following assumption is made for the function $f$.

ASSUMPTION 1. The sign of $\partial f / \partial u_{k}$ is known, and $\partial f / \partial u_{k} \neq 0, \forall\left(x, u_{k}\right) \in \Omega_{x} \times \mathbb{R}$, where $\Omega_{x} \subset \mathbb{R}^{n}$ is a certain controllability region containing the origin.

The control objective is to design a robust control for the system such that the output is stabilized at the origin, and meanwhile all the signals in the closed-loop system remain semi-globally uniformly ultimately bounded (SGUUB).

REMARK 1. Assumption 1 implies that $\partial f / \partial u_{k}$ is strictly either positive or negative. Without lose of generality, assume that $\partial f / \partial u_{k}>0, \forall\left(x, u_{k}\right) \in \Omega_{x} \times \mathbb{R}$. Assumption 1 is reasonable because $\partial f / \partial u_{k}$ being away from zero is controllable condition of system (1).

REMARK 2. In practice, many engineering plants including active magnetic bearing systems [3], vibrating systems $[15]$, continuous stirred-tank reactor systems [16], etc, are reasonably described in the non-affine forms (1). Because, for these plants, they are difficult to be exactly described in affine forms even though the modeling errors are neglected.

REMARK 3. Under Assumption 1, the non-affine nonlinear system described by (1) includes a large class of nonlinear systems. It is should be noted that many elegant results in this area have been obtained using the approximators (e.g., NN) to estimate the uncertainties. Next section in this paper will be presented a novel dynamic linear filter which also can be used to estimate the uncertainties. Thus, compared with NN control, the proposed control architecture is quite simple.

\section{ROBUST OUTPUT FEEDBACK CONTROL DESIGN}

By adding and subtracting $g u$ in the right-hand side of system (1), feedback linearization is performed by

$$
y_{k+n}=g u_{k}+\Delta_{k},
$$

where $g>0$ is a design constant, and

$$
\Delta_{k}=f\left(x_{k}, u_{k}\right)-g u_{k},
$$

which is the feedback linearization error. Let the control input be determined as

$$
u_{k}=\frac{1}{g}\left(u_{d, k}+u_{\Delta, k}\right),
$$

where $u_{d, k}$ is a dynamic feedback controller designed to stabilize linearized dynamics in (2) by assuming $\Delta_{k}=0 ; u_{\Delta, k}$ is a filterbased compensator designed to handle the effect of $\Delta_{k}$, i.e., given a small parameter $\varepsilon>0$, find a $u_{\Delta, k}$ such that

$$
\left|u_{\Delta, k}+\Delta_{k}\right| \leq \varepsilon, \forall k \geq k_{0},
$$

where $k_{0} \in \mathbb{Z}_{0}^{+}$

Due to the bijection $\psi: u_{k} \rightarrow f\left(\cdot, u_{k}\right)$, it is noted that the compensator $u_{\Delta, k}$ is designed to deal with $\Delta_{k}$, whereas $\Delta_{k}$ is a function of $u_{\Delta, k}$ through $f\left(\cdot, u_{k}\right)$ and $u_{k}$. Like the result in the continuous time [5, 17], the following lemma is introduced to guarantee the existence and uniqueness of a solution of

$$
h\left(x_{k}, u_{d, k}, u_{\Delta, k}\right)=0
$$

where $h\left(x_{k}, u_{d, k}, u_{\Delta, k}\right)=u_{\Delta, k}+\Delta_{k}$.

LEMma 2. 5. 17 Let constant $g>0$ satisfies the following inequalities

$$
0<\frac{1}{2}\left(\frac{\partial f}{\partial u_{k}}\right)<g, \forall\left(x, u_{k}\right)
$$

then (6) has a unique solution over the entire input domain of interest.

\subsection{Dynamic feedback controller design}

Under the assumption that $\Delta_{k}=0$, then we can let $u_{\Delta, k}=0$ and design $u_{d, k}$. System (2) reduce as

$$
y_{k+n}=u_{d, k}
$$

which can be stabilized using classical linear control design, such as the dynamic feedback control

$$
\begin{aligned}
\xi_{k+1} & =A_{d} \xi_{k}+B_{d} y_{k}, \\
u_{d, k} & =C_{d}^{T} \xi_{k}+d_{d} y_{k},
\end{aligned}
$$

where $A_{d} \in \mathbb{R}^{(n-1) \times(n-1)}, B_{d}, C_{d} \in \mathbb{R}^{n-1}$ and $d_{d} \in \mathbb{R}$ are appropriately chosen parameters such that matrix $A_{s}=$ $\left[\begin{array}{cc}A+d_{d} B C^{T} & B C_{d}^{T} \\ B_{d} C^{T} & A_{d}\end{array}\right]$ is Schur, i.e., the spectral radius $\sigma\left(A_{s}\right)<$ 
It should be noticed that it is required $\operatorname{dim} \xi_{k} \geq n-1$ due to the dynamics in (8) having $n$ poles at the origin [18]. Without lose of generality, the minimum dimension is chosen in the sequel. And it is the same reason for considering the dimension of a filter given in the next subsection.

\subsection{Compensator design}

Substituting (4) and (9b) into (2), we have

$$
y_{k+n}=u_{d, k}+u_{\Delta, k}+\Delta_{k},
$$

To compensate the effect of $\Delta_{k}$, we construct a filter by using the available output

$$
\begin{aligned}
\hat{y}_{k+n} & =-\epsilon d_{c}\left(y_{k}-\hat{y}_{k}\right)-C_{c}^{T} \eta_{k}+u_{d, k}+u_{\Delta, k}, \\
\epsilon^{-1} \eta_{k+1} & =A_{c} \eta_{k}+\epsilon B_{c}\left(y_{k}-\hat{y}_{k}\right),
\end{aligned}
$$

where $\eta_{k} \in \mathbb{R}^{n-1}, u_{\Delta, k}$ will be designed in 14 and $\epsilon$ is a small parameter to be specified later. By introducing the variable

$$
\tilde{y}_{k}=\epsilon\left(y_{k}-\hat{y}_{k}\right),
$$

and from $(10)$ and $(11)$, we have

$$
\begin{aligned}
& \epsilon^{-1} \tilde{y}_{k+n}=d_{c} \tilde{y}_{k}+C_{c}^{T} \eta_{k}+\Delta_{k}, \\
& \epsilon^{-1} \eta_{k+1}=A_{c} \eta_{k}+B_{c} \tilde{y}_{k},
\end{aligned}
$$

where $A_{c} \in \mathbb{R}^{(n-1) \times(n-1)}, B_{c}, C_{c} \in \mathbb{R}^{n-1}$ and $d_{c}, \epsilon \in \mathbb{R}$ are appropriately chosen parameters such that not only matrix $A_{s s}=$ $\left[\begin{array}{cc}A+d_{c} B C^{T} & B C_{c}^{T} \\ B_{c} C^{T} & A_{c}\end{array}\right]$ is Schur, but also $\tilde{y}_{k}$ converges in a faster time scale than $y_{k}$.

Observing (13a), the compensator is designed as

$$
u_{\Delta, k}=d_{c} \tilde{y}_{k}+C_{c}^{T} \eta_{k} .
$$

from which and combining (13a) the left-hand side of (5) becomes

$$
\begin{aligned}
& \left|u_{\Delta, k}+\Delta_{k}\right| \\
= & \left|d_{c} \tilde{y}_{k}+C_{c}^{T} \eta_{k}+\Delta_{k}\right|=\epsilon^{-1}\left|\tilde{y}_{k+n}\right| .
\end{aligned}
$$

Theorem analysis in the next subsection will be shown that, under the proposed controller (4, , 9) and (14) and some conditions, the output $y_{k}$ and $\epsilon^{-1} \tilde{y}_{k+n}$ converge to the origin and all the signals in the closed-loop system remain bounded.

REMARK 4. In the literatures [4] 18], observers were constructed to estimate the unmeasurable states, then the NNs were employed to approximate the uncertainties. The novel filter proposed in this paper is built based on the available output information and can be directly estimated the uncertainties without using the NNs.

\subsection{Stability analysis}

For clarity, let

$$
\begin{gathered}
\tilde{x}_{k}=\left[\tilde{y}_{k}, \tilde{y}_{k+1}, \cdots, \tilde{y}_{k+n}\right]^{T}, \\
X_{k}=\left[x_{k}^{T}, \xi_{k}^{T}\right]^{T}, \tilde{X}_{k}=\left[\tilde{x}_{k}^{T}, \eta_{k}^{T}\right]^{T}, \\
\bar{B}=\left[\begin{array}{c}
B \\
0
\end{array}\right], I_{\epsilon}=\left[\begin{array}{cc}
I_{(n-1) \times(n-1)} & 0 \\
0 & \epsilon I_{n \times n}
\end{array}\right], \\
\sigma_{A s}=\sigma\left(A_{s}\right), \sigma_{A s s}=\sigma\left(A_{s s}\right), \sigma_{\epsilon}=\sigma\left(I_{\epsilon}\right)
\end{gathered}
$$

$$
F_{x, k}\left(x_{k}, u_{k}\right)=\frac{\partial f\left(x_{k}, u_{k}\right)}{\partial x_{k}}, f_{u, k}\left(x_{k}, u_{k}\right)=\frac{\partial f\left(x_{k}, u_{k}\right)}{\partial u_{k}}
$$

The compact form of system 13 can be written by

$$
I_{\epsilon}^{-1} \tilde{X}_{k+1}=A_{s s} \tilde{X}_{k}+\bar{B} \Delta_{k}
$$

Substituting (4), (9) and (14) into (2), the closed-loop takes the compact form of

$$
X_{k+1}=A_{s} X_{k}+\bar{B} \bar{B}^{T} \Xi_{k}
$$

where $\Xi_{k}=A_{s s} \tilde{X}_{k}+\bar{B} \Delta_{k}$

From the mean value theorem, there exists a $\theta \in(0,1)$ such that

$$
f\left(x_{k+1}, u_{k+1}\right)-f(0,0)=F_{x, k+1}^{\theta} x_{k+1}+f_{u, k+1}^{\theta} u_{k+1}
$$

where $F_{x, k+1}^{\theta}=F_{x, k+1}\left(\theta x_{k+1}, \theta u_{k+1}\right), \quad f_{u, k+1}^{\theta}=$ $f_{u, k+1}\left(\theta x_{k+1}, \theta u_{k+1}\right)$.

Combining (3), (16) and (18), we have

$$
\begin{aligned}
\Xi_{k+1} & =A_{s s} \tilde{X}_{k+1}+\bar{B} \Delta_{k+1} \\
& =A_{s s} I_{\epsilon} \Xi_{k}+\bar{B}\left[f\left(x_{k+1}, u_{k+1}\right)-g u_{k+1}\right] \\
& =A_{s s} I_{\epsilon} \Xi_{k}+\bar{B}\left[F_{x, k+1}^{\theta} x_{k+1}+\left(f_{u, k+1}^{\theta}-g\right) u_{k+1}\right] \\
& =A_{s s} I_{\epsilon} \Xi_{k}+\alpha X_{k+1}+\beta \tilde{X}_{k+1} \\
& =\bar{A}_{s s} I_{\epsilon} \Xi_{k}+\alpha\left[A_{s} X_{k}+\bar{B} \bar{B}^{T} \Xi_{k}\right]
\end{aligned}
$$

where

$$
\begin{aligned}
& \alpha=\bar{B}\left[F_{x, k+1}^{\theta}+\frac{f_{u, k+1}^{\theta}-g}{g} d_{d} C^{T}, \frac{f_{u, k+1}^{\theta}-g}{g} C_{d}^{T}\right] \\
& \beta=\frac{f_{u, k+1}^{\theta}-g}{g} \bar{B}\left[d_{c} C^{T}, C_{c}^{T}\right] \\
& \bar{A}_{s s}=A_{s s}+\beta .
\end{aligned}
$$

The following theorem shows that the proposed controller stabilizes systems (1) under the assumption that the feedback linearization error $\Delta_{k} \equiv 0$.

THEOREM 3. Under the assumption that $\Delta_{k} \equiv 0$, consider the closed-loop consisting of system (1) satisfying Assumption 1, the filter (11), and the controller (4), (9) and (14). Then, there exists an $\epsilon^{*}>0$ such that for all $\epsilon \in\left(0, \epsilon^{*}\right)$, the closed-loop system is globally exponential asymptotic stable.

Proof. Combining (17) and (19), we have

$$
\left[\begin{array}{c}
X_{k+1} \\
\Xi_{k+1}
\end{array}\right]=\Phi_{1}\left[\begin{array}{l}
X_{k} \\
\Xi_{k}
\end{array}\right]
$$

where $\Phi_{1}=\left[\begin{array}{cc}A_{s} & \bar{B} \bar{B}^{T} \\ 0 & A_{s s} I_{\epsilon}\end{array}\right]$. From linear system theory, we know that the dynamics 20 is stable if and only if $\sigma\left(\Phi_{1}\right)<1$, i.e., $\sigma_{A s} \sigma_{A s s} \sigma_{\epsilon}<1$. It is easy to obtain that the system is globally exponential asymptotic stable for all $\epsilon \in\left(0, \epsilon_{1}^{*}\right)$, where $\epsilon_{1}^{*}=$ $1 /\left(\sigma_{A s} \sigma_{A s s}\right)$.

Based on Assumption 1 the following theorem shows the proposed controller stabilizes systems (1) despite the presence of $\Delta_{k}$.

THEOREM 4. Consider the closed-loop consisting of system (1) satisfying Assumption 1, the filter (11), and the controller (4), (9) and (14) and let $\epsilon^{*}=1 /\left(\sigma_{A s} \sigma_{A s s}\right)$. For all $x(0) \in \Omega_{x}$ and all $\epsilon \in\left(0, \epsilon^{*}\right)$ which satisfies

$$
\sigma_{A s}+\sigma_{A s s} \epsilon+c_{1} c_{2}<\sqrt{2}
$$


then the system output $y_{k}$ converges to the origin and all signals in the closed-loop system remain $S G U U B$, where $c_{1}$ is an upper boundedness of $\alpha$, i.e., $\|\alpha\| \leq c_{1}$ and $c_{2}=\left\|\bar{B} \bar{B}^{T}\right\|$.

Proof. Observing the matrix $\bar{A}_{s s}$ defined in 19 . Then there exists a dialog matrix

$$
M=\left[\begin{array}{ccc}
I_{(n-1) \times(n-1)} & 0 & 0 \\
0 & \frac{f_{u, k+1}^{\theta}}{g} & 0 \\
0 & 0 & I_{(n-1) \times(n-1)}
\end{array}\right]
$$

such that

$$
\bar{A}_{s s}=A_{s s} M .
$$

From Assumption 1 we have

$$
0<\frac{f_{u, k+1}^{\theta}}{g}<\frac{1}{2} .
$$

Thus, $\sigma(M)=1$. Moreover, since $f$ is a continuously differetiable with respect to all the arguments and $\Omega_{x}$ is a compact set and Assumption 1 then $\partial F / \partial x_{k}$ and $\partial f / \partial u_{k}$ are bounded. i.e., there exist a constant $c_{1}>0$ such that $\|\alpha\| \leq c_{1}$.

Combining (17), 19] and 22, we have

$$
\begin{aligned}
\left\|X_{k+1}\right\| & \leq \sigma_{A s}\left\|X_{k}\right\|+c_{2}\left\|\Xi_{k}\right\| \\
\left\|\Xi_{k+1}\right\| & \leq\left(\sigma_{A s s} \sigma_{\epsilon}+c_{1} c_{2}\right)\left\|\Xi_{k}\right\|+c_{1} \sigma_{A s}\left\|X_{k}\right\|
\end{aligned}
$$

The compact form of 23] and (23) can be written by

$$
\left[\begin{array}{l}
\left\|X_{k+1}\right\| \\
\left\|\Xi_{k+1}\right\|
\end{array}\right] \leq \Phi_{2}\left[\begin{array}{l}
\left\|X_{k}\right\| \\
\left\|\Xi_{k}\right\|
\end{array}\right]
$$

where $\Phi_{2}=\left[\begin{array}{cc}\sigma_{A s} & c_{2} \\ c_{1} \sigma_{A s} & \sigma_{A s s} \sigma_{\epsilon}+c_{1} c_{2}\end{array}\right]$. If $\sigma\left(\Phi_{2}\right)<1$, then the dynamics 25 is stable. In what follows, we make analysis of the matrix $\Phi_{2}$.

(i)If the design parameter $1<\epsilon<1 /\left(\sigma_{A s} \sigma_{A s s}\right)$, then $\sigma_{\epsilon}=\epsilon$ and

$$
\Phi_{2}=\left[\begin{array}{cc}
\sigma_{A s} & c_{2} \\
c_{1} \sigma_{A s} & \sigma_{A s s} \epsilon+c_{1} c_{2}
\end{array}\right] .
$$

Furthermore

$$
\sigma\left(\Phi_{2}\right)=\frac{\left(\sigma_{A s}+\sigma_{A s s} \epsilon+c_{1} c_{2}\right)^{2}}{2}-\sigma_{A s} \sigma_{A s s} \epsilon
$$

From 26, when $\epsilon$ satisfies 21] then $\sigma\left(\Phi_{2}\right)<1$.

(ii)If $\epsilon \leq 1$, then $\sigma_{\epsilon}=1$. Clearly, when

$$
\sigma_{A s}+\sigma_{A s s}+c_{1} c_{2}<\sqrt{2}
$$

we remain have $\sigma\left(\Phi_{2}\right)<1$.

REMARK 5. Unlike the previous control approaches using $N N$ and/or fuzzy systems [4, 5, 17], the robust output tracking controller proposed here is easy to implement due to its linear control architecture. The parameters in the proposed control have clear physical meanings and the desired dynamic performance can be achieved by tuning the design parameters.

\section{CONCLUSION}

The main contribution of this paper has presented robust design for a general class of nonlinear systems non-affine in control by output feedback. A novel high-gain filter has been constructed to generate a fast time-scale signal to estimate the modeling errors without using NNs and/or fuzzy systems. Compared with the previous adaptive controllers, the proposed controller is of great significance in engineering practice due to its linear control architecture, high dynamic performance and clear physical meanings. In the future, investigation on a general class of nonaffine nonlinear systems will be interesting research topics in this field.

\section{ACKNOWLEDGEMENTS}

Supported by National Natural Science Foundation of China (61374003), Foundation for Distinguished Young Talents in Higher Education of Guangdong Province of China (2014KQNCX173), Doctor scientific research of Guangdong Polytechnic Normal University, Project of Guangdong Polytechnic Normal University (14KJY12), Foundation for 1st batch Distinguished Talents in Higher Education of Guangdong Province of China, 2013'.

\section{REFERENCES}

[1] Chen Z. F., Ge S. S., Zhang Y., Li Y. 2014. Adaptive neural control of MIMO nonlinear systems with a block-triangular pure-feedback control structure. IEEE Transactions on Neural Networks and Learning Systems. 25(11), 2017-2029.

[2] Chen Z. F., Zhang Y. 2014. Robust control of a class of nonaffine nonlinear systems by state and output feedback. Journal of Central South University, 21(4), 1322-1328.

[3] Hsu C. T., Chen S. L. 2003. Nonlinear control of a 3-pole active magnetic bearing system. Automatica. 39, 291-298.

[4] Ge S. S., Hang C. C., Zhang T. 1999. Adaptive neural network control of nonliear systems by state and output feedback. IEEE Transactions on Systems, Man, and CyberneticsPart B: Cybernetics. 29(6), 818-828.

[5] Park J. H., Kim S.H. 2004. Direct adaptive output-feedback fuzzy controller for a nonaffine nonlinear system. IEE Proceedings Control Theory and Applications. 51(1), 65-72.

[6] Wang C., Hill D. J., Ge S. S., Chen G. 2006. An ISS-modular approach for adaptive neural control of pure-feedback systems. Automatica. 42, 723-731.

[7] Song Y., Grizzle J. W. 1993. Adaptive Output-Feedback Control of a Class of Discrete-Time Nonlinear Systems. In Proceedings of American Control Conference. 1359-1364.

[8] Kanellakopoulos I. 1994. A discrete-time adaptive nonlinear system. IEEE Transactions on Automatic Control. 39(11), $2362-2365$

[9] Chen F. C., Khalil H. K. 1995, Adaptive control of a class of nonlinear discrete-time systems using neural networks. IEEE Transactions on Automatic Control. 40(5), 791C801.

[10] Jagannathan S., Lewis F. L. 1996. Discrete-time neural net controller for a class of nonlinear dynamical systems. IEEE Transactions on Automatic Control. 41(11), 1693C1699.

[11] Ge S. S., Li G. Y., Lee T. H. Adaptive NN control for a class of strict-feedback discrete-time nonlinear systems. Automatica, $39,807-819$.

[12] Ge S. S., Yang C., Lee T. H. 2008. Adaptive predictive control using neural network for a class of pure-feedback systems in discrete time. IEEE Transactions on Neural Networks. 19(9), 1599-1614. 
[13] Liu Y. J., Chen P., Wen G. X., Tong S. 2011. Adaptive Neural output feedback tracking control for a class of uncertain discrete-time nonlinear systems. IEEE Transactions on Neural Networks. 22(7), 1162-1167.

[14] Ge S. S., Wang C. 2002. Adpative NN control of uncertain nonlinear pure-feedback systems. Automatica. 38, 671-682.

[15] Shiriaev, A. S., Ludvigsen, H., Egeland, O., Fradkov, A. L. 1999. Swinging up of non-affine in control pendulum. In Proceedings of American Control Conference, San Diego, California, USA, 4039-4044.

[16] Ge S. S., Lee T. H., Harris C. J. 1998. Adaptive Neural Network Control of Robotic Manipulators. London, U.K.: World Scientific.

[17] Park J. H., Huh S. H., Kim S.H., Seo S. J., Park G. T. 2005. Direct adaptive controller for nonaffine nonlinear systems using self-structuring neural networks. IEEE Transactions on Neural Networks. 16(2), 414-422.

[18] Hovakimyan N., Nardi F., Calise A. J. 2002. A novel error boserver-based adaptive output feedback aproach for control of uncertain systems. IEEE Transactions on Automatic Control. 47(8), 1310-1314. 